ing from a clinical standpoint. It is reminiscent of the oft-noted clinical findings in hypertrophic cardiomyopathy wherein the degree of diastolic impairment is often described as "out of proportion" to the observed ventricular hypertrophy, thus suggesting a significant myocellular contribution to disease pathogenesis. It is interesting to speculate that the wellknown salutary effects of postinfarction treatment with metoprolol may reflect the molecular findings in the current paper. Thus the results presented by Perrino et al. lay the groundwork for a robust 2-pronged therapeutic approach based on relief of the inciting pathogenic stimulus and direct treatment of the underlying molecular derangements, perhaps via novel compounds to displace active PI3K isoforms from the receptor complex.

Address correspondence to: Jil C. Tardiff, Albert Einstein College of Medicine, 1300 Morris Park Avenue, Ullmann Building, Room 316, Bronx, New York 10461, USA. Phone: (718) 430-8914; Fax: (718) 4308819; E-mail: tardiff@aecom.yu.edu.
1. Meerson, F.Z. 1962. Compensatory hyperfunction of the heart and cardiac insufficiency. Circ. Res. 10:250-258.

2. Lorell, B.H., Apstein, C.S., Weinberg, E.O., and Cunningham, M.J. 1990. Diastolic function in left ventricular hypertrophy: clinical and experimental relationships. Eur. Heart J. 11(Suppl. G):54-64

3. Ho, K.K., Pinsky, J.L., Kannel, W.B., and Levy, D. 1993. The epidemiology of heart failure: the Framingham Study. J. Am. Coll. Cardiol. 22:6A-13A.

4. Raskoff, W.J., Goldman, S., and Cohn, K. 1976. The "athletic heart". Prevalence and physiological significance of left ventricular enlargement in distance runners. JAMA. 236:158-162.

5. Bevegard, B.S., and Shepherd, J.T. 1967. Regulation of the circulation during exercise in man. Physiol. Rev. 47:178-213.

6. Giusti, R., Bersohn, M.M., Malhotra, A., and Scheuer, J. 1978. Cardiac function and actomyosin ATPase activity in hearts of conditioned and deconditioned rats. J. Appl. Physiol. 44:171-174.

7. Wilkins, B.J., et al. 2004. Calcineurin/NFAT coupling participates in pathological, but not physiological, cardiac hypertrophy. Circ. Res. 94:110-118.

8. Barki-Harrington, L., Perrino, C., and Rockman, H.A. 2004. Network integration of the adrenergic system in cardiac hypertrophy. Cardiovasc. Res. 63:391-402.

9. Perrino, C., et al. 2006. Intermittent pressure overload triggers hypertrophy-independent cardiac dysfunction and vascular rarefaction. J. Clin. Invest. 116:1547-1560. doi:10.1172/JCI25397.

10. Shioi, T., et al. 2000. The conserved phosphoinositide 3-kinase pathway determines heart size in mice. EMBO J. 19:2537-2548.
11. Frey, N., et al. 2004. Mice lacking calsarcin-1 are sensitized to calcineurin signaling and show accelerated cardiomyopathy in response to pathological biomechanical stress. Nat. Med. 10:1336-1343.

12. Ertz-Berger, B.R., et al. 2005. Changes in the chemi$\mathrm{cal}$ and dynamic properties of cardiac troponin $\mathrm{T}$ cause discrete cardiomyopathies in transgenic mice. Proc. Natl. Acad. Sci. U. S. A. 102:18219-18224.

13. Dorn, G.W., 2nd, and Force, T. 2005. Protein kinase cascades in the regulation of cardiac hypertrophy. J. Clin. Invest. 115:527-537. doi:10.1172/ JCI200524178.

14. Nienaber, J.J., et al. 2003. Inhibition of receptor-localized PI3K preserves cardiac $\beta$-adrenergic receptor function and ameliorates pressure overload heart failure. J. Clin. Invest. 112:1067-1079. doi:10.1172/JCI200318213.

15. Naga Prasad, S.V., Esposito, G., Mao, L., Koch, W.J., and Rockman, H.A. 2000. Gbetagamma-dependent phosphoinositide 3-kinase activation in hearts with in vivo pressure overload hypertrophy. J. Biol. Chem. 275:4693-4698.

16. Crackower, M.A., et al. 2002. Regulation of myocardial contractility and cell size by distinct PI3KPTEN signaling pathways. Cell. 110:737-749.

17. Mann, D.L. 2005. Left ventricular size and shape: determinants of mechanical signal transduction pathways. Heart Fail. Rev. 10:95-100.

18. Knoll, R., et al. 2002. The cardiac mechanical stretch sensor machinery involves a $Z$ disc complex that is defective in a subset of human dilated cardiomyopathy. Cell. 111:943-955.

19. Devereux, R.B., et al. 2004. Prognostic significance of left ventricular mass change during treatment of hypertension. JAMA. 292:2350-2356.

\title{
Mechanisms of sympathoadrenal failure and hypoglycemia in diabetes
}

\author{
Philip E. Cryer \\ Division of Endocrinology, Metabolism and Lipid Research, General Clinical Research Center, and Diabetes Research and Training Center, \\ Washington University School of Medicine, St. Louis, Missouri, USA.
}

\begin{abstract}
A reduced sympathoadrenal response, induced by recent antecedent hypoglycemia, is the key feature of hypoglycemia-associated autonomic failure (HAAF) and, thus, the pathogenesis of iatrogenic hypoglycemia in diabetes. Understanding of the mechanism(s) of that reduced response awaits new insight into its basic molecular, cellular, organ, and whole-body physiology and pathophysiology in experimental models. In this issue of the JCI, McCrimmon and colleagues report that application of urocortin I (a corticotrophin-releasing factor receptor-2 agonist) to the ventromedial hypothalamus reduces the glucose counterregulatory response to hypoglycemia in rats (see the related article beginning on page 1723). Thus, hypothalamic urocortin I release during antecedent hypoglycemia is, among other possibilities, a potential mechanism of HAAF.
\end{abstract}

Nonstandard abbreviations used: HAAF, hypoglycemia-associated autonomic failure; T1DM, type 1 diabetes mellitus; VMH, ventromedial hypothalamus.

Conflict of interest: The author has served on advisory boards of Novo Nordisk Inc., Takeda Pharmaceuticals North America Inc., and MannKind Corp. in recent years. Citation for this article: J. Clin. Invest. 116:1470-1473 (2006). doi:10.1172/JCI28735.
The clinical problem of iatrogenic hypoglycemia in people with diabetes mellitus (1) has stimulated bidirectional translational research. That included, initially, patientoriented research based on the then-existing body of knowledge and, more recently, basic research into the molecular, cellular, organ, and whole-body physiology of glucose counterregulation - the mechanisms that normally prevent or rapidly correct hypoglycemia - and its pathophysiology in experimental models (2). The latter is exemplified by the report from McCrimmon and colleagues (3) in this issue of the JCI. In the aggregate, such basic research will undoubtedly lead to more informative studies in humans and, hopefully, the ultimate elimination of hypoglycemia from the lives of people with diabetes.

The clinical problem is clear to millions of people with diabetes and, increasingly, to their caregivers: iatrogenic hypoglycemia is the limiting factor in the glycemic management of diabetes $(1,2)$. It causes recurrent morbidity in most people with type 1 diabetes mellitus (T1DM) and many with $\mathrm{T} 2 \mathrm{DM}$ and is sometimes fatal. The barrier 


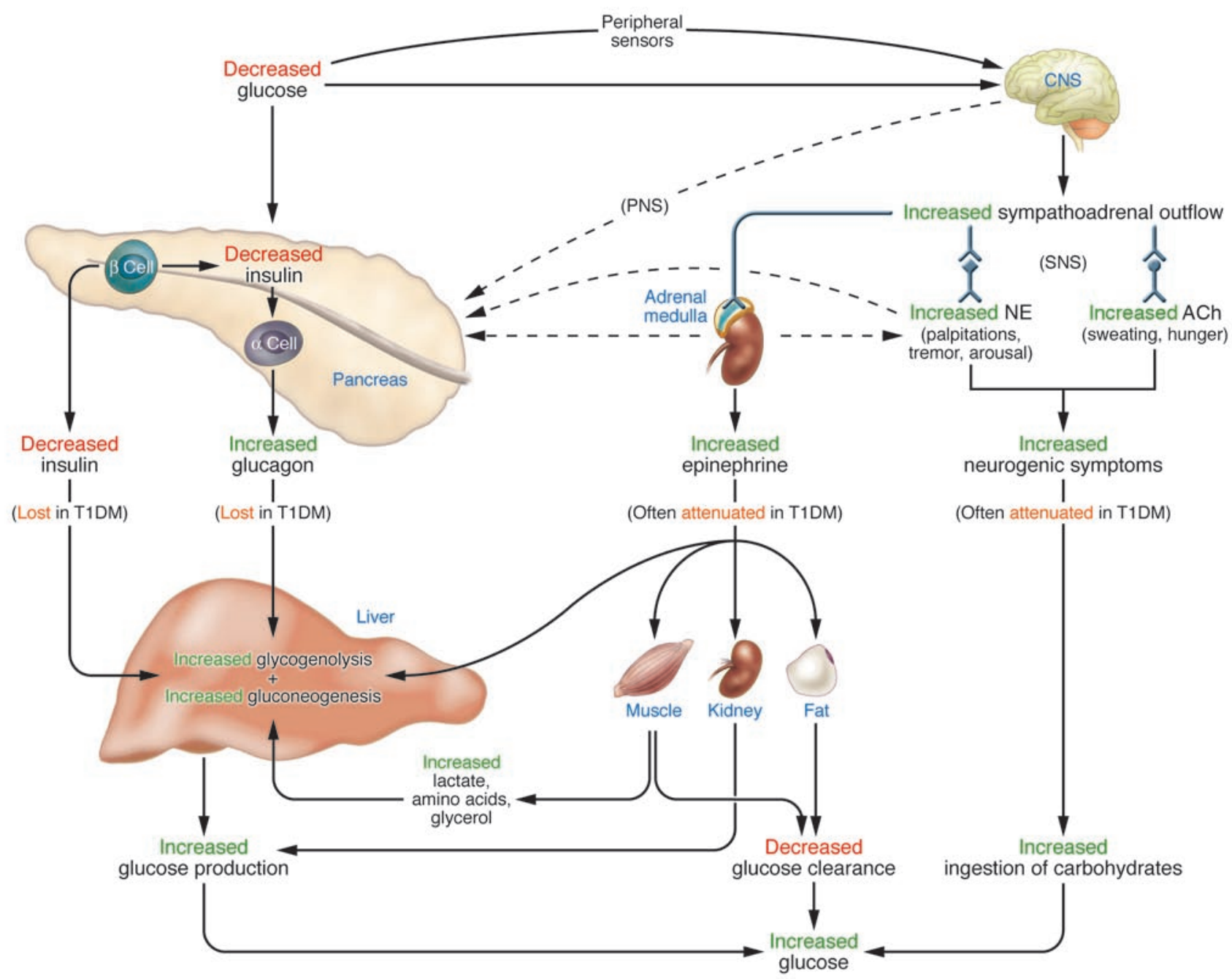

Figure 1

Physiological and behavioral defenses against hypoglycemia. Decrements in insulin and increments in glucagon are lost and increments in epinephrine and neurogenic symptoms are often attenuated in insulin-deficient - T1DM and advanced T2DM — diabetes. SNS, sympathetic nervous system; PNS, parasympathetic nervous system; NE, norepinephrine; ACh, acetylcholine; $\alpha$ cell, pancreatic islet $\alpha$ cells; $\beta$ cell, pancreatic islet $\beta$ cells.

of hypoglycemia precludes maintenance of euglycemia over a lifetime of diabetes and, thus, full realization of the now wellestablished vascular benefits of glycemic control $(4,5)$. Even asymptomatic episodes of hypoglycemia impair defenses against subsequent hypoglycemia by causing hypoglycemia-associated autonomic, specifically sympathoadrenal, failure and thus a vicious cycle of recurrent hypoglycemia.

\section{Compromised defenses against hypoglycemia}

The physiological defenses against falling plasma glucose concentrations (Figure 1) include: (a) decreased pancreatic islet $\beta$ cell insulin secretion; (b) increased pancreatic islet $\alpha$ cell glucagon secretion; and, absent the latter, (c) increased adrenomedullary epi- nephrine secretion (6). The behavioral form of defense (Figure 1) is the ingestion of food prompted by symptoms of hypoglycemia (6). All of these defenses are compromised $(1,2)$ in T1DM (7) and advanced T2DM (8). This involves both peripheral (pancreatic islet) and CNS alterations. Both decrements in insulin and increments in glucagon are lost. The former is the result of $\beta$ cell failure. The latter is plausibly attributed to loss of the decrement in intra-islet insulin that normally signals increased glucagon secretion (9-11). In the setting of absent insulin and glucagon responses, attenuated epinephrine responses cause the clinical syndrome of defective glucose counterregulation that is associated with a 25 -fold or greater increased risk of severe hypoglycemia $(1,2)$. Attenuated sympathoadrenal, largely sym- pathetic neural (12), responses also cause the clinical syndrome of hypoglycemia unawareness that, by compromising the behavioral defense, is also associated with an increased risk of severe hypoglycemia (1, 2). Thus, hypoglycemia is the result of the interplay of absolute or relative (to exogenous glucose delivery, endogenous glucose production, or insulin sensitivity) therapeutic insulin excess and compromised glucose counterregulation $(1,2,13)$.

\section{Hypoglycemia-associated autonomic failure}

The concept of hypoglycemia-associated autonomic failure (HAAF) in T1DM (7) and advanced T2DM (8) (Figure 2) posits that recent antecedent iatrogenic hypoglycemia causes both defective glucose counterregu- 


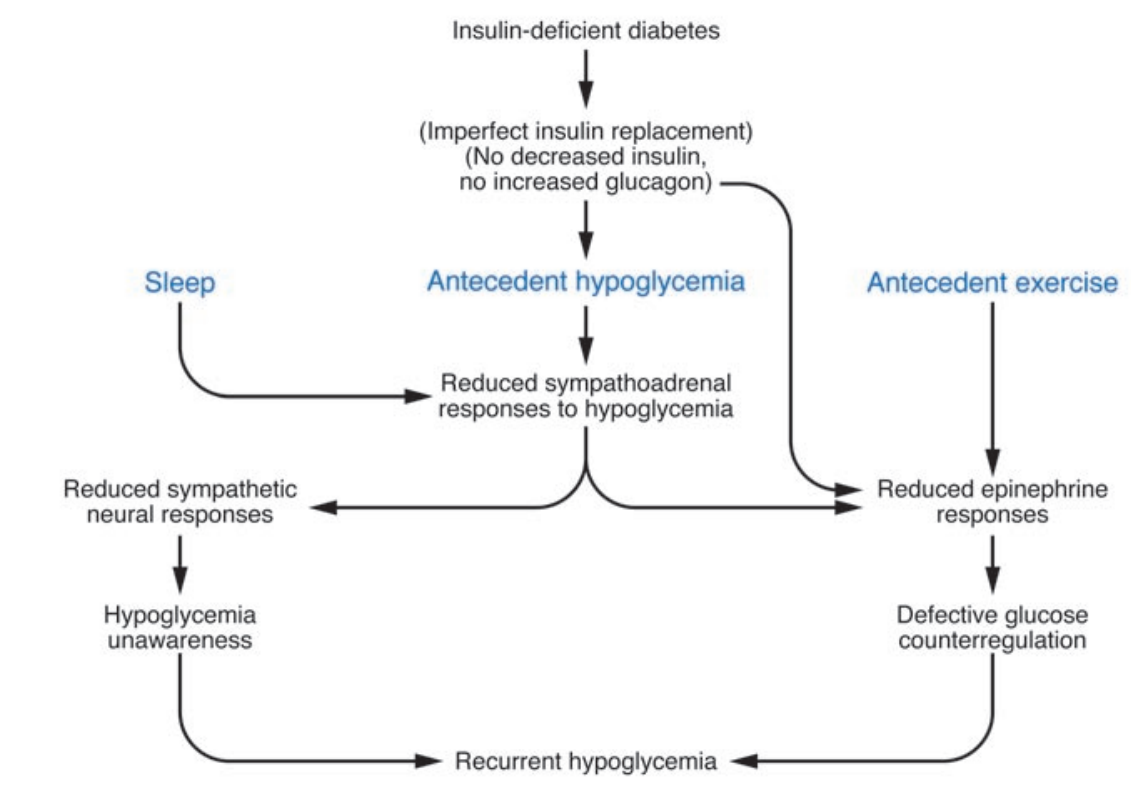

Figure 2

HAAF in T1DM and advanced T2DM. Figure modified from the New England Journal of Medicine, with permission from the Massachusetts Medical Society (1).

lation and hypoglycemia unawareness and, thus, a vicious cycle of recurrent hypoglycemia $(1,2)$. It causes defective glucose counterregulation by reducing epinephrine responses to a given level of subsequent hypoglycemia in the setting of absent decrements in insulin and increments in glucagon. It causes hypoglycemia unawareness by reducing sympathoadrenal and the resulting neurogenic symptom responses to a given level of subsequent hypoglycemia. Sleep and antecedent exercise cause a similar phenomenon (1) (Figure 2). The clinical impact of HAAF, including the finding that hypoglycemia unawareness and the reduced epinephrine component of defective glucose counterregulation are reversed by as little as $2-3$ weeks of scrupulous avoidance of hypoglycemia in most affected patients, is well established $(1,2)$. However, the mechanism(s) of the key feature of HAAF, the attenuated sympathoadrenal response to falling plasma glucose concentrations, are unknown (2).

The shift of the glycemic thresholds for sympathoadrenal responses to lower plasma glucose concentrations caused by recent antecedent hypoglycemia (or by sleep or prior exercise) could be the result of alterations in the peripheral afferent or efferent components of the autonomic nervous system or within the CNS (2). Much of the recent research has focused on the latter, especially the hypothalamus $(2,3)$. However, hypoglycemia activates wide- spread brain regions including the medial prefrontal cortex (14).

The potential CNS mechanisms of the reduced sympathoadrenal response, and thus of HAAF, have been reviewed (2). In my view (2), the balance of evidence, including more recent evidence (15), weighs heavily against the systemic mediator (e.g., cortisol) hypothesis and the brain fuel (e.g., glucose) transport hypothesis, although increased transport of other fuels remains to be explored fully (16). Thus, assuming a primary $\mathrm{CNS}$ alteration, the global brain metabolism hypothesis is the most attractive. The array of potential mechanisms within the brain (reviewed in ref. 2) range from posthypoglycemic increased glucokinase activity in critical hypothalamic neurons (17) to posthypoglycemic brain glycogen supercompensation (18). In addition, alterations of ATP-sensitive $\mathrm{K}^{+}$channel function, AMPactivated protein kinase activity, $\gamma$-amino butyric acid release, insulin signaling, and expression of angiotensinogen and related genes as well as of paraventricular nucleus activity, cerebral glucose metabolism, and cerebral blood flow have been proposed (2). The findings of McCrimmon and colleagues (3) raise another possibility. They report that ventromedial hypothalamus (VMH) application of urocortin I, a corticotrophin-releasing factor receptor-2 agonist, suppresses the counterregulatory response to hypoglycemia (an effect that lasted for at least 24 hours), whereas that of cortico- trophin-releasing factor, a predominant corticotrophin-releasing factor receptor-1 agonist, amplifies that response in rats. Urocortin I was also shown to alter the glucose sensitivity of $\mathrm{VMH}$ glucose-sensing neurons in whole-cell current clamp experiments in brain slices. Thus, increased hypothalamic urocortin I release during antecedent hypoglycemia could explain a decreased sympathoadrenal response to subsequent hypoglycemia. However, that causal connection was not demonstrated and, therefore, remains a provocative theoretical possibility.

Ultimately, the problem of hypoglycemia (and that of hyperglycemia) in diabetes will likely be solved by the development of safe and effective methods that provide plasma glucose-regulated insulin replacement or secretion. That will probably involve closed-loop insulin replacement (online glucose sensor-computer interface-insulin infusion pump) until implantation of glucose-responsive, insulin-secreting cells becomes feasible for widespread use. Pending that, there is clear a need for new insight into the fundamental mechanisms of the physiology of glucose counterregulation and its pathophysiology in experimental models that leads to clinical strategies that are then shown to both reduce the risk of hypoglycemia and facilitate glycemic control in people with diabetes (i.e., bidirectional translational research).

\section{Acknowledgments}

The author's cited work was supported, in part, by US Public Health Service/NIH grants R37 DK27085, MO1 RR00036, P60 DK20579, and T32 DK07120 and by a fellowship award from the American Diabetes Association. Janet Dedeke prepared this manuscript.

Address correspondence to: Philip E. Cryer, Campus Box 8127, Washington University School of Medicine, 660 South Euclid Avenue, St. Louis, Missouri 63110, USA. Phone: (314) 362-7635; Fax: (314) 3627989; E-mail: pcryer@wustl.edu.

\footnotetext{
1. Cryer, P.E. 2004. Diverse causes of hypoglycemiaassociated autonomic failure in diabetes. N. Engl. J. Med. 350:2272-2279.

2. Cryer, P.E. 2005. Mechanisms of hypoglycemiaassociated autonomic failure and its component syndromes in diabetes. Diabetes. 54:3592-3601.

3. McCrimmon, R.J., et al. 2006. Corticotrophin-releasing factor receptors within the ventromedial hypothalamus regulate hypoglycemia-induced hormonal counterregulation. J. Clin. Invest. 116:1723-1730. doi:10.1172/JCI27775.

4. The Diabetes Control and Complications Trial Research Group. 1993. The effect of intensive
} 
treatment of diabetes on the development and progression of long-term complications in insulin-dependent diabetes mellitus. N. Engl. J. Med. 329:977-986

5. The Diabetes Control and Complications Trial/ Epidemiology of Diabetes Interventions and Complications (DCCT/EDIC) Study Research Group. 2005. Intensive diabetes treatment and cardiovascular disease in patients with type 1 diabetes. N. Engl. J. Med. 353:2643-2653.

6. Cryer, P.E. 2001. The prevention and correction of hypoglycemia. In Handbook of physiology: a critical, comprehensive presentation of physiological knowledge and concepts. Section 7, The endocrine system. Volume 2, The endocrine pancreas and regulation of metabolism. L.S. Jefferson and A.D. Cherrington, editors. Oxford University Press. New York, New York, USA. 1057-1092.

7. Dagogo-Jack, S.E., Craft, S., and Cryer, P.E. 1993. Hypoglycemia-associated autonomic failure in insulin-dependent diabetes mellitus: recent ante- cedent hypoglycemia reduces autonomic responses to, symptoms of, and defense against subsequent hypoglycemia. J. Clin. Invest. 91:819-828.

8. Segel, S.A., Paramore, D.S., and Cryer, P.E. 2002 Hypoglycemia-associated autonomic failure in advanced type 2 diabetes. Diabetes. 51:724-733.

9. Raju, B., and Cryer, P.E. 2005. Loss of the decrement in intraislet insulin plausibly explains loss of the glucagon response to hypoglycemia in insulindeficient diabetes. Diabetes. 54:757-764.

10. Gosmanov, N.R., et al. 2005. Role of the decrement in intraislet insulin for the glucagon response to hypoglycemia in humans. Diabetes Care. 28:1124-1131.

11. Israelian, Z., et al. 2005. Increasing the decrement in insulin secretion improves glucagon responses to hypoglycemia in advanced type 2 diabetes. Diabetes Care. 28:2691-2696.

12. DeRosa, M.A., and Cryer, P.E. 2004. Hypoglycemia and the sympathoadrenal system: neurogenic symptoms are largely the result of sympathetic neural, rather than adrenomedullary, activation.
Am. J. Physiol. Endocrinol. Metab. 287:E32-E41.

13. Cryer, P.E., Davis, S.N., and Shamoon, H. 2003. Hypoglycemia in diabetes. Diabetes Care. 26:1902-1912.

14. Teves, D., Videen, T.O., Cryer, P.E., and Powers, W.J. 2004. Activation of human medial prefrontal cortex during autonomic responses to hypoglycemia. Proc. Natl. Acad. Sci. U. S. A. 101:6217-6221.

15. Goldberg, P.A., et al. 2006. Antecedent hypercortisolemia is not primarily responsible for generating hypoglycemia-associated autonomic failure. Diabetes. 55:1121-1126.

16. Mason, G.F., Petersen, K.F., Lebon, V., Rothman, D.L., and Shulman, G.I. 2006. Increased brain monocarboxylic acid transport and utilization in type 1 diabetes. Diabetes. 55:929-934.

17. Kang, L., et al. 2006. Glucokinase is a critical regulator of ventromedial hypothalamic neuronal glucosensing. Diabetes. 55:412-420.

18. Choi, I.-Y., Seaquist, E.R., and Gruetter, R. 2003. Effect of hypoglycemia on brain glycogen metabolism in vivo. J. Neurosci. Res. 72:25-32.

\title{
IPEX and the role of FOXP3 in the development and function of human Tregs
}

\author{
Séverine Le Bras and Raif S. Geha
}

Division of Immunology, Children's Hospital, and Department of Pediatrics, Harvard Medical School, Boston, Massachusetts, USA.

\begin{abstract}
Genetic defects in the transcription factor forkhead box protein P3 (FOXP3) cause immune dysregulation, polyendocrinopathy, enteropathy, $X$-linked (IPEX). IPEX is thought to be due to a defect in naturally arising $\mathrm{CD4}^{+}$Tregs. In this issue of the JCI, Bacchetta and colleagues demonstrate that patients with IPEX and missense mutations in FOXP3 provide insight into the role of various domains of FOXP3 in the development and function of Tregs (see the related article beginning on page 1713).
\end{abstract}

In the past decade, naturally arising $\mathrm{CD} 4^{+} \mathrm{CD} 25^{+}$Tregs have emerged as being critical for the maintenance of peripheral immunological tolerance (1). Neonatal thymectomy in mice and thymic hypoplasia in humans (DiGeorge syndrome) result in impaired Treg generation and in the development of organ-specific autoimmune diseases $(2,3)$. Treg generation in the thymus requires interaction with MHC class II molecules expressed by cortical epithelial cells (4). Other signals have also been implicated, including those delivered by CD28, CD40 ligand, and the cytokines TNF- $\alpha$ and TGF- $\beta$ (5). However, IL-2, which is necessary for Treg survival

Nonstandard abbreviations used: FKH, forkhead; FOXP3, forkhead box protein P3; GITR, glucocorticoidinduced TNF receptor; IPEX, immune dysregulation, polyendocrinopathy, enteropathy, X-linked.

Conflict of interest: The authors have declared that no conflict of interest exists.

Citation for this article: J. Clin. Invest. 116:1473-1475 (2006). doi:10.1172/JCI28880. in the periphery, is dispensable for their development $(6,7)$.

Tregs represent a small subset of $\mathrm{CD}^{+} \mathrm{T}$ cells (5-10\%). They express several surface markers including CD25 (the IL-2 receptor $\alpha$-chain), CTL-associated antigen 4 (CTLA4), and glucocorticoid-induced TNF receptor (GITR). However, these molecules are upregulated in naive $\mathrm{CD} 4^{+} \mathrm{CD} 25^{-} \mathrm{T}$ cells following TCR stimulation and cannot therefore serve as absolute Treg markers. Recently, several groups identified the forkhead family transcription factor forkhead box protein P3 (FOXP3) as a specific molecular marker of Tregs $(8,9)$. FOXP3 expression seems to be largely restricted to a small subset of TCR $\alpha \beta$ T cells and defines 2 pools of Tregs with suppressive activity: $\mathrm{CD} 4^{+} \mathrm{CD} 25^{\text {high }}$ cells and a minor population of $\mathrm{CD} 4{ }^{+} \mathrm{CD} 25^{\text {low }}-$ $\mathrm{T}$ cells. Thus, FOXP3 expression correlates better with suppressor activity than CD25 expression (10). Ectopic expression of Foxp3 in vitro and in vivo is sufficient to convert naive murine $\mathrm{CD}^{+} \mathrm{T}$ cells to Tregs (11).
In contrast, overexpression of FOXP3 in human $\mathrm{CD}^{+} \mathrm{CD} 25^{-} \mathrm{T}$ cells in vitro is not sufficient to generate potent suppressor activity, suggesting that additional factors are required for bona fide Treg activity (12). Foxp3 expression cannot be readily induced in murine $\mathrm{T}$ cells by stimulation in vitro. In humans, cross-linking of the TCR and CD28 stimulation, or antigen-specific stimulation, can induce $\mathrm{CD}^{+} \mathrm{CD}^{+} 5^{-} \mathrm{FOXP}^{-}$cells to become Tregs and express FOXP3 and exert suppressor function $(11,13)$. These results suggest that de novo generation of Tregs may be a natural consequence of the immune response in humans.

Tregs are anergic in vitro. They fail to proliferate and secrete IL-2 in response to TCR ligation, and they depend on IL-2 generated following activation of $\mathrm{CD} 4^{+} \mathrm{CD} 25^{-} \mathrm{T}$ cells to survive and exert their function. An in vitro assay that measures the ability of $\mathrm{CD} 4^{+} \mathrm{CD} 25^{+}$ $\mathrm{T}$ cells to suppress $\mathrm{CD} 4^{+} \mathrm{CD} 25^{-} \mathrm{T}$ cell proliferation is commonly used to assess Treg function (14). The mechanisms mediating the suppression of immune responses in vivo by Tregs are still unclear. Several studies support the involvement of contact-dependent inhibition, while others suggest that Tregs can exert their function by secreting immunosuppressive cytokines such as IL-10 or TGF- $\beta$ or by directly killing their target in a perforin-dependent manner (15). 